\title{
Discussing the future of amphibians in research
}

\author{
Report of the NC3Rs/ZSL Workshop on Amphibian Welfare.
}

\author{
Samuel Brod, Lola Brookes and Trenton W. J. Garner
}

A mphibians have been used as experimental organisms for centuries. Recent years have seen a renewed interest in amphibians as models of human development and disease and an urgent need to understand and mitigate the impacts of the chytridiomycotan and ranaviral pathogens that pose a global threat to these animals. Alongside this increase in laboratory use comes an increased responsibility to conduct research using amphibians with proper consideration made of the unique welfare requirements of this diverse vertebrate class. Despite this, knowledge of the welfare needs of amphibians remains limited, with little scientifically justified guidance or evidencebased refinements for their captive care ${ }^{1-4}$.

On $5^{\text {th }}$ October 2017 the National Centre for the Replacement, Refinement and Reduction of Animals in Research (NC3Rs) and the Zoological Society of London (ZSL) jointly hosted a workshop to discuss the welfare of amphibians in research. This workshop brought together experts from academia and the zoo community to identify the key topics in amphibian welfare and identify the next steps that can be taken to help monitor and improve conditions for amphibians in research. The workshop included 72 participants, with specializations ranging from oncology and developmental biology to conservation and epidemiology. The following report summarises the key outcomes of the day and recommendations for research priorities in this area.

\section{Summary of workshop talks}

The demand for Xenopus laevis (Fig. 1) and $X$. tropicalis as models for disease, development and genetic manipulation has led to an increase in their use and distribution worldwide. The coordinator of the European Xenopus Resource Centre (EXRC; https://xenopusresource.org/), Professor Matthew Guille (University of Portsmouth, UK), discussed the welfare and biosecurity challenges created by the storage and distribution of hundreds of genetically modified Xenopus lines. By improving current methods of sperm recovery and cryopreservation, his centre has significantly reduced the number of male frogs required for research, refined their husbandry,

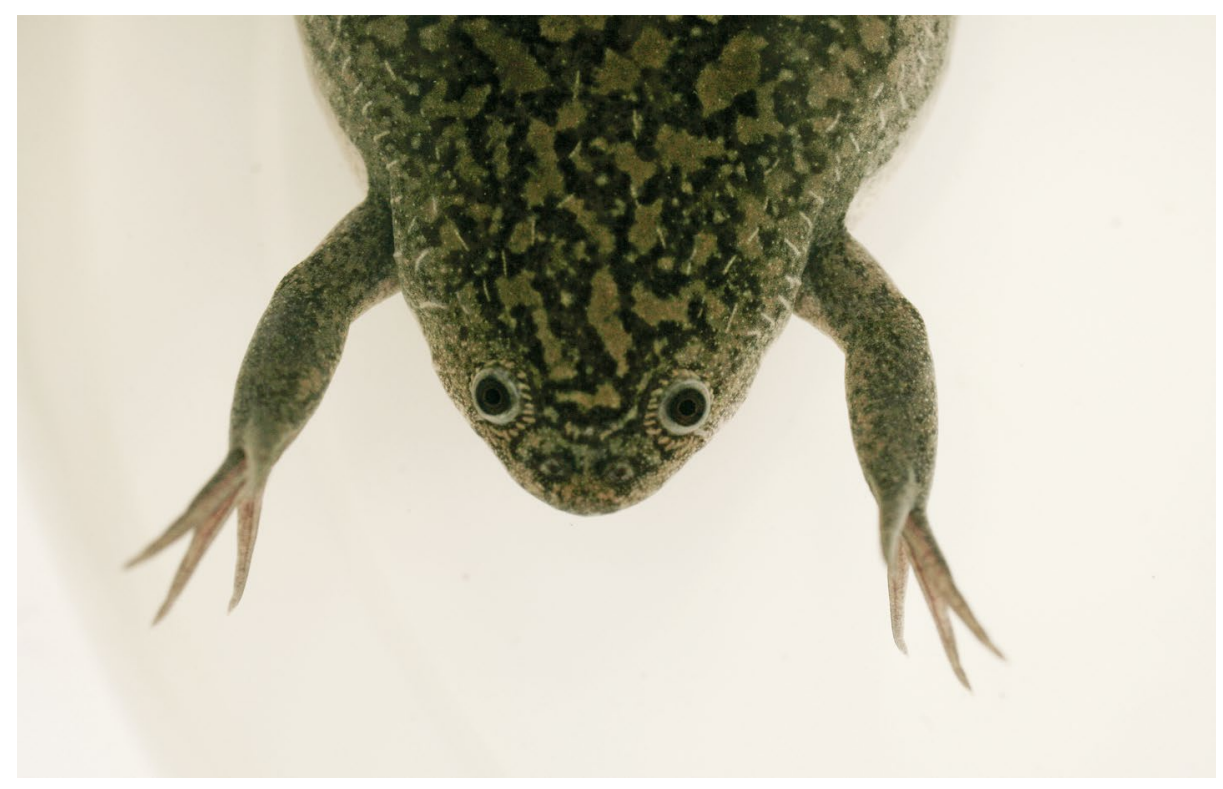

Fig. 1 | Adult Xenopus laevis. Credit: Xenopus Resource Centre, Portsmouth, UK

and potentially eliminated the need for transporting male frogs ${ }^{5}$. Complementing Professor Guille's talk, Professor Jacques Robert (University of Rochester Medical Center, USA) described how the rapid increase in Xenopus use in laboratories has highlighted the absence of realistic, scientifically based welfare standards for these animals. Professor Robert aided in the update and standardization of the recently published Cold Spring Harbor Protocols guidelines for Xenopus husbandry ${ }^{6}$.

Assessing the welfare of captive amphibians has proven notoriously difficult. Few behavioural or physiological indicators of welfare have been developed or shown to be effective; those that do exist cannot be consistently applied across amphibian species. Dr Lottie Hosie (University of Chester, UK) reported her lab's efforts to validate such methods, again focusing on Xenopus laevis. She demonstrated that a combination of behavioural (such as activity levels and tank butting) and endocrine (waterborne corticosterone) measures of stress may prove useful in gauging amphibian welfare, but that greater effort and collaboration by researchers is needed to confirm their more general application and to develop additional indicators ${ }^{7,8}$.

Professor Richard Griffiths (University of Kent, UK) outlined the challenges facing effective research on amphibians in the field as well as the limited and sometimes restrictive welfare guidelines that govern their use in scientific procedures (for examples, ${ }^{9,10}$ ). These animals have a diverse set of morphologies, habitats and physiological needs, so a one-size-fits-all approach for field work with amphibians seems untenable. Building on this topic, Professor Caren Helbing (University of Victoria, Canada) presented her work developing transcriptomic- and metabolomic-focused sampling methodologies that allow health and population information on multiple amphibian species to be obtained from water samples taken from their habitat. ${ }^{11}$. Validation of these non-lethal, non-invasive methodologies may pave the way for better assessment of the impact of environmental change, pollutants and infection on amphibian welfare in both the laboratory and field, as well as help to implement the reduction and refinement principles of the 3Rs. 


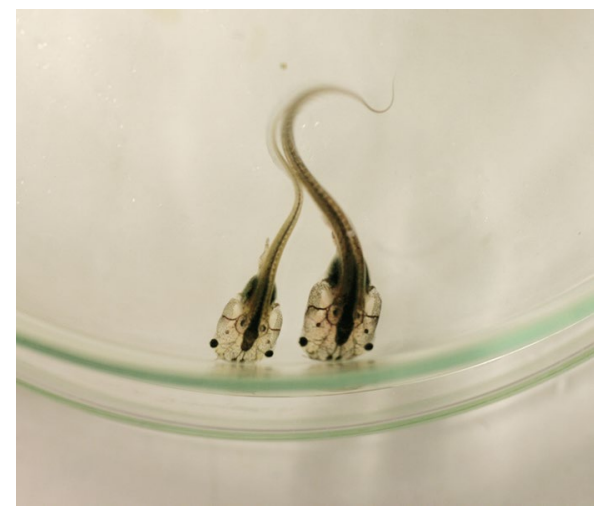

Fig. 2 | Larval Xenopus laevis. Credit: Xenopus Resource Centre, Portsmouth, UK

Live infection studies are currently the only effective means of studying the ranavirus epidemic threatening amphibian populations worldwide. Dr Stephen Price (University College London, UK) presented an alternative in vitro model using amphibian cell culture systems to study host-pathogen interactions ${ }^{12}$. Such research stands to significantly reduce animal use while helping combat this serious disease.

Perhaps the greatest practical challenge to increasing scientifically supported amphibian welfare is securing the funding to support the research. Dr Mark Prescott (NC3Rs, UK) closed off the talks by drawing on NC3Rs' experience of funding 3Rs-relevant science, including the aforementioned work of Guille and Hosie, and highlighted how to write a successful grant application to the NC3Rs and integrate an interest in welfare with wider research aims.

\section{Summary of breakout sessions}

Talks were followed by afternoon breakout sessions that identified subjects of broad importance for improving amphibian welfare in a research setting:

\section{The dual problem of multiple species} and life history stages. In contrast to many other vertebrate classes, dozens of amphibian species are currently used in research, with diverse and specific housing and husbandry requirements. Compounding this issue, most amphibians have complex life histories, with significantly different housing and husbandry needs as they mature from embryo to larvae to juvenile to adult $^{2}$ (Fig. 2). These issues were regarded as the major impediment to devising a basic, transferrable set of welfare and husbandry standards for amphibians.

One proposed alternative was to select a "flagship species" to serve as the platform for initiating a concerted effort to optimize amphibian welfare. The obvious choice would be the most commonly used amphibian in laboratory research, Xenopus laevis. However, others commented that such a course would only serve to recapitulate the welfare limitations, as the ecology and natural history of Xenopus species do not represent the majority of frog or toad species; even less so the newts, salamanders and caecilians (Fig. 3).

The need for basic, relevant guidelines on amphibian care and husbandry. Despite accepting that the requirements of different species and life history stages cannot be harmonized into a single set of guidelines, the participants still recognized that fundamental guidance on the housing and husbandry of amphibians is markedly limited compared to that of other taxa. Most representatives regarded the evidence base for existing guidance to be dubious, dated or not truly applicable to amphibians. Some suggested that regulators can lack the appropriate knowledge base to decide the suitability of housing, husbandry and validated measures of welfare and humane endpoints for amphibians. The distinction between different jurisdictional oversights for zoo, laboratory and conservation-based research also proved a point of contention; some researchers felt unfairly restricted in the work they may carry out while others fall outside the lines of governance ${ }^{13}$. Participants also acknowledged a lack of interaction amongst the different sectors, which impairs the collaborative development of husbandry protocols.

There was a consensus that current guidelines and best practice documents on amphibian housing and husbandry existing training resources specific to amphibian welfare should be updated, used in regulator-approved training courses, and delivered by more training centers. However, there was also some uncertainty regarding how widely and to whom these need to be revised and expanded and that

new guidelines should apply. This hesitation again derived from current uncertainties regarding jurisdictional oversight for amphibian research in the field and in zoos and conservation centers.

The need for basic measures of welfare across the amphibian research community. Workshop participants generally agreed that current means of measuring welfare in amphibians are poor and sometimes contradictory across research groups and countries. Many of the measures in use are inefficient, not validated or indiscriminately applied across amphibian species that exhibit divergent behaviours and responses to stress and poor health. These issues were attributed at least in part to a perceived poor understanding of the need to apply the 3Rs in amphibian research, poor communication between amphibian research groups, a lack of funding for welfare research and the challenges created by using multiple species of amphibians in research.

\section{A call for an active community on amphibian welfare in research.}

The need for better communication on welfare between amphibian researchers was a common theme. The creation of a community to actively discuss the topic of, and share findings on, amphibian welfare would help to harmonise best practice in husbandry, drive new research and bring to the fore any new developments in the field.

Alongside this call for better communication came a desire for the creation of a centralized resource hub on amphibian care, husbandry and breeding. Some of the suggested purposes for this hub would be to:

- Create an outlet to publish husbandry and welfare information

- Build a centralised resource on welfare for building consensus

- Aid in cross sector learning

- Improve information sharing and knowledge exchange

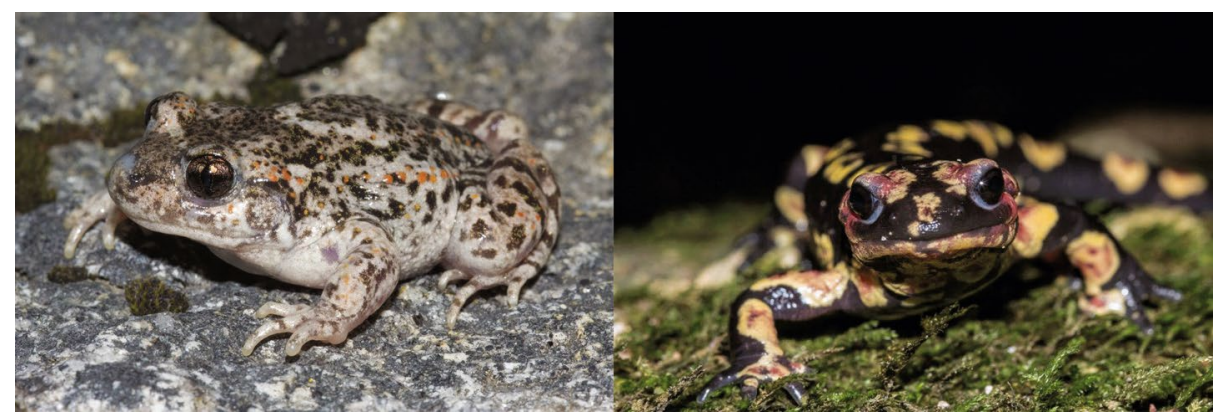

Fig. 3 | A midwife toad (left) and fire salamander (right). Credit: G.M. Rosa, Zoological Society of London 


\section{Table 1 | Some important but unresolved questions on amphibian welfare}

1 Is MS222 an appropriate chemical for euthanasia of amphibians?

2 What biomarkers of welfare are applicable to amphibians?

3 What is the efficacy of current analgesia and anaesthesia?

$4 \quad$ Can Xenopus superovulation / general breeding protocols be refined?

5 Are corticosterone readouts valid measures of welfare?

6 Can established preference tests be created for amphibians?

7 What non-invasive monitoring methodologies exist for these animals?

8 Have species-specific baseline behaviours been documented?

9 What is the ideal nutritional composition of a regulated amphibian diet?

10 Is live food a necessity in an amphibian diet?

11 How does water quality and composition affect amphibian welfare?

12 What (if any) are the health benefits of ultraviolet light for amphibians?

13 Does providing a gradient in the physical captive environment of amphibians (to enable choice of microenvironment) improve health and welfare?

\section{Securing funding for research on} amphibian welfare. Amphibian research appears to be in a transitionary period, with some optimistic that current innovations in genome editing will drive a continued interest in the species while others felt amphibian science to be in decline. The reticence of zoos and other conservation organisations to be associated with research that involves invasive, regulated procedures on animals limits the progress of amphibian research. At the breakout group focused on securing funding for welfare research, participants suggested that this issue stems partly from a lack of cohesion between amphibian researchers, which limits their ability to construct project proposals of the necessary size and scope to attract large funders. Greater efforts to integrate welfare research into grants ostensibly focused on basic research as a source of "added value" would likely aid in getting more welfare research published.
While much of the day focused on highlighting the shortcomings of amphibian welfare in laboratories, the consensus was that the workshop had been productive. There was clear enthusiasm for greater research on amphibian welfare and agreement that the workshop had revealed key challenges that must be faced for the field to progress. In addition to these issues, several important unanswered questions facing amphibian researchers were identified throughout the day, which are summarised in Table 1. Together, these five challenges and thirteen questions could serve as the stepping stones towards greater engagement with this topic and the advancement of amphibian welfare.

\footnotetext{
Samuel Brod ${ }^{1 *}$, Lola Brookes ${ }^{2}$ and Trenton W. J. Garner 2,3,4

${ }^{1}$ National Centre for the Replacement, Refinement and Reduction of Animals in Research (NC3Rs),
}

London, UK. ${ }^{2}$ Institute of Zoology, Regent's Park, London, UK. ${ }^{3}$ Unit for Environmental Sciences and Management, North-West University, Private Bag x6001, Potchefstroom 2520, South Africa. ${ }^{4}$ Non-profit Association Zirichiltaggi - Sardinia Wildlife Conservation, Strada Vicinale Filigheddu 62/C, I-07100 Sassari, Italy.

*e-mail:Samuel.Brod@nc3rs.org.uk

Published online: 5 November 2018

https://doi.org/10.1038/s41684-018-0193-6

\section{References}

1. National Resource Council. Guide for the care and use of laboratory animals. (National Academies Press, Washington, DC, 2010).

2. Harvey Pough, F. ILAR J 48, 203-213 (2007).

3. Reed, B. Guidanace on the housing and care of the African clawed frog Xenopus laevis. (Royal Society for the Prevention of Cruelty to Animals, Horsham, United Kingdom, 2005). Report.

4. Tinsley, R. Amphibians, with special reference to Xenopus. The UFAW handbook on the care and management of laboratory and other research animals, 741-760 (2010).

5. Pearl, E. et al. Theriogenology 92, 149-155 (2017).

6. Vleminckx, Kris, ed. Xenopus Methods and Protocols. pp. 1-17 (Humana Press, New York, NY 2018).

7. Holmes, A. M. et al. Appl Anim. Behav. Sci. 185, 131-136 (2016).

8. Holmes, A. M., Emmans, C. J., Coleman, R., Smith, T. E. \& Hosie, C. A. Gen Comp. Endocrinol 266, 21-28 (2018).

9. Paul, E., Sikes, R. S., Beaupre, S. J. \& Wingfield, J. C. ILAR J 56, 312-334 (2016).

10. Perry, G., Wallace, M. C., Perry, D., Curzer, H. \& Muhlberger, P. J Herpetol 45, 547-555 (2011).

11. Campbell, L. J. et al. Mol Ecol. 27, 1413-1427 (2018).

12. Price, S.J. et al. bioRxiv, 272369 (2018).

13. Sikes, R. S., Paul, E. \& Beaupre, S. J. BioScience 62, 830-834 (2012).

\section{Acknowledgements}

We would like to thank each of the speakers at the event for their advice in putting this report together. We are also grateful to Dr Mark Prescott (NC3Rs) for his helpful comments on the manuscript.

\section{Competing interests}

Samuel Brod is a member of the NC3Rs, the organisation that funded and co-hosted the event reported upon. Lola Brookes and Trenton WJ Garner are members of the Zoological Society of London, the organisation that co-hosted the event reported upon. 